\title{
Contents
}

\section{Modelling and Simulation Theory}

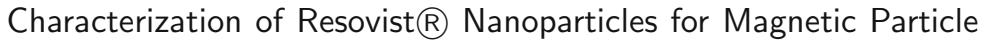

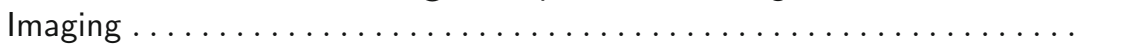

Takashi Yoshida, Keiji Enpuku, Frank Ludwig, Jan Dieckhoff,

Thilo Wawrzik, Aidin Lak, Meinhard Schilling

Nonlinear Behavior of Magnetic Fluid in Brownian Relaxation:

Numerical Simulation and Derivation of Empirical Model .............

Takashi Yoshida, Keiji Enpuku

Magnetic Particle Imaging Using Ferromagnetic Magnetization

Stephan Euting, Fernando M. Araújo-Moreira, Waldemar Zylka

Magnetic Particle Imaging: Exploring Particle Mobility

Thilo Wawrzik, Frank Ludwig, Meinhard Schilling

System Calibration Unit for Magnetic Particle Imaging: Focus Field Based System Function .............................

Aleksi Halkola, Thorsten Buzug, Jürgen Rahmer, Bernhard Gleich, Claas Bontus

\section{Spectroscopy}

Characterization of Magnetic Nanoparticles for Magnetic Particle Imaging by Magnetorelaxometry, AC Susceptibility, Magnetic Particle Spectroscopy and Static Magnetization Measurements ...

Frank Ludwig, Thilo Wawrzik, Meinhard Schilling

Perspectives of Magnetic Particle Spectroscopy for Magnetic Nanoparticle Characterization ..........................

Thilo Wawrzik, Meinhard Schilling, Frank Ludwig 
Initial MPS Rsponse of Adsorptively-Coated Fluorescent Iron Oxide

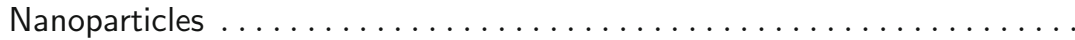

Jabadurai Jayapaul, Daniel Truhn, Fabian Kiessling,

Kerstin Lüdtke-Buzug, Thorsten M. Buzug

Evaluation of Different Magnetic Particle Systems with Respect to Its MPI Performance .............................

Dietmar Eberbeck, Lutz Trahms, Harald Kratz

Determination of System Functions for Magnetic Particle Imaging . . . . . .

Matthias Graeser, Sven Biederer, Mandy Grüttner, Hanne Wojtczyk,

Timo F. Sattel, Wiebke Tenner, Gael Bringout, Thorsten M. Buzug

\section{Magnetic Particle Separation}

Microfluidic System as a Tool for Magnetic Separation of Human Cells with Diagnostic Relevance..........................

J. Schemberg, J. Bertram, M. Hottenrott, T. Legler, S. Howitz,

K. Lemke, G. Gastrock, J. Metze

Potential of Improving MPI Performance by Magnetic Separation . . . . . . .

N. Löwa, D. Eberbeck, U. Steinhoff, F. Wiekhorst, L. Trahms

\section{Magnetic Nanoparticles}

Fractionated Magnetic Multicore Nanoparticles for Magnetic Particle Imaging

Silvio Dutz, Dietmar Eberbeck, Robert Müller, Matthias Zeisberger

Precision Synthesis of Iron Oxide Nanoparticles and Their Use as Contrast Agents

Jan Niehaus, Sören Becker, Christian Schmidtke, Katja Werner, Horst Weller

Synthesis of Single-Core Iron Oxide Nanoparticles as a Tracer for Magnetic Particle Imaging.

Aidin Lak, Thilo Wawrzik, Frank Ludwig, Meinhard Schilling

New Perspectives for MPI: A Toolbox for Tracer Research ............

Nicole Gehrke, Andreas Briel, Frank Ludwig, Hilke Remmer,

Thilo Wawrzik, Stefan Wellert

Superparamagnetic Iron Oxide Nanoparticles: Evaluation of Stability of SPIONs in Different Milieu for Magnetic Particle Imaging

Kerstin Lüdtke-Buzug, Céline Borchers 
3D Semi-quantification of Nanoparticle Content in Tissue on

Experimental and Commercial $\mu \mathrm{CT}$-Scanner................... 111

Helene Rahn, Katharina Bayer, Stefan Odenbach, Stefan Lyer,

Christoph Alexiou, Frank Wiekhorst, Lutz Trahms, Michael Baumann,

Julia Buckwar, Mechthild Krause

Biomaterials for Regenerative Medicine: Cytotoxicity of

Superparamagnetic Iron Oxide Nanoparticles in Stem Cells . . . . . . . . . . 117

D. Schneider, K. Lüdtke-Buzug

Tracer Development for Magnetic Particle Imaging . . . . . . . . . . . . . . 123

Harald Kratz, Dietmar Eberbeck, Susanne Wagner, Jörg Schnorr,

Matthias Taupitz

The Potential of Magnetic Particle Imaging in the Competitive

Environment of Cardiac Diagnostics . . . . . . . . . . . . . . . . . . 129

Gunnar Schütz

Iron Oxide Nanoparticles - Tracer for Magnetic Particle Imaging . .

Ulrich Pison, Cordula Grüttner, Fritz Westphal, Barbara Kleiner,

Nicole Barthel, Katharina Roepke

\section{Magnetic Particle Imaging Theory}

Experimental Evaluation of Correlation-Based Image Reconstruction

Method for Magnetic Particle Imaging. . . . . . . . . . . . . . . . . . . . 143

Takumi Honma, Yasutoshi Ishihara

Relaxation in x-space Magnetic Particle Imaging . . . . . . . . . . . . . . . . 149

Laura R. Croft, Patrick Goodwill, Matt Ferguson, Kannan Krishnan,

Steven Conolly

Linear and Shift Invariance of Magnetic Particle Imaging . . . . . . . . . . . 155

Kuan Lu, Patrick Goodwill, Steve Conolly

Efficient Positioning of the Field-Free Point in Magnetic Particle

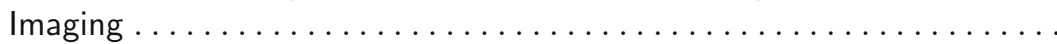

Tobias Knopp, Timo F. Sattel, Thorsten M. Buzug

Visualization of Instruments in Interventional Magnetic Particle Imaging (iMPI): A Simulation Study on SPIO Labelings . . . . . . . . . . . . . . . 167 Hanne Wojtczyk, Julian Haegele, Mandy Grüttner, Wiebke Tenner, Gael Bringout, Matthias Graeser, Florian M. Vogt, Jörg Barkhausen, Thorsten M. Buzug 


\section{Medical Applications}

Red Blood Cells as Magnetic Carriers for MPI Applications . . . . . . . . . 175

A. Antonelli, C. Sfara, M. Magnani, J. Rahmer, B. Gleich, J. Borgert,

J. Weizenecker

Ex Vivo Magnetic Sentinel Lymph Node Detection in Colorectal Cancer

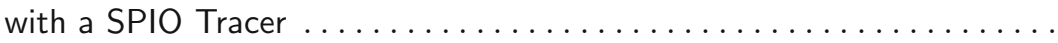

Joost J. Pouw, Raluca M. Fratila, Aldrik H. Velders, Bennie ten Haken, Quentin A. Pankhurst, Joost M. Klaase

Distribution of Superparamagnetic Nanoparticles in Lymphatic Tissue for Sentinel Lymph Node Detection in Breast Cancer by Magnetic Particle Imaging

Dominique Finas, Kristin Baumann, Katja Heinrich, Britta Ruhland, Lotta Sydow, Ksenija Gräfe, Timo Sattel, Kerstin Lüdtke-Buzug, Thorsten Buzug

Behavior of Superparamagnetic Iron Oxides in Magnetic Targeting Models.

loana Slabu, Anjali Roeth, Gernot Güntherodt, Thomas Schmitz-Rode, Martin Baumann

Diagnostic Imaging in Cancer Therapy with Magnetic Nanoparticles .... . .

Stefan Lyer, Rainer Tietze, Stephan Dürr, Tobias Struffert,

Tobias Engelhorn, Marc Schwarz, Arnd Dörfler, Lubos Budinsky,

Andreas Hess, Wolfgang Schmidt, Roland Jurgons, Christoph Alexiou

Engineering Contrast Agents for Gastro-Intestinal Magnetic Particle Imaging: The Biological Perspective. .

Katrin Ramaker, Niels Röckendorf, Andreas Frey

Visualization of Instruments for Cardiovascular Intervention Using MPI

Julian Haegele, Jürgen Rahmer, Bernhard Gleich, Claas Bontus, Jörn Borgert, Hanne Wojtczyk, Thorsten M. Buzug, Jörg Barkhausen, Florian M. Vogt

Efficient Encoding Methods for Small Numbers of Pixels to Achieve High Sensitivity for Screening John B. Weaver 


\section{Magnetic Particle Imaging}

Influence of Magnetic Field Optimization on Image Quality Achieved for Efficient Radon-Based Reconstruction in Field Free Line Imaging in

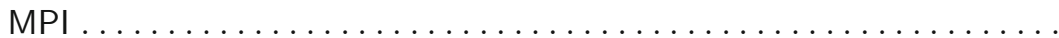

Marlitt Erbe, Tobias Knopp, Timo F. Sattel, Thorsten M. Buzug

Slicing Frequency Mixed Traveling Wave for 3D Magnetic Particle Imaging

P. Vogel, M.A. Rückert, P. Klauer, W.H. Kullmann, P.M. Jakob, V.C. Behr

Magnetic-Particle-Imaging for Sentinel Lymph Node Biopsy in Breast Cancer . . . . . . . . . . . . . . . . . . . . . . . . . . . . . .

Ksenija Gräfe, Timo F. Sattel, Kerstin Lüdtke-Buzug, Dominique Finas, Jörn Borgert, Thorsten M. Buzug

Experimental 3D X-Space Magnetic Particle Imaging Using Projection Reconstruction ................................ 243 Justin Konkle, Patrick Goodwill, Oscar Carrasco-Zevallos, Steven Conolly

Enlarging the Field of View in Magnetic Particle Imaging - A Comparison . . . . . . . . . . . . . . . . . . . . . . . . . 249 Mandy Grüttner, Timo F. Sattel, Matthias Graeser, Hanne Wojtczyk, Gael Bringout, Wiebke Tenner, Thorsten M. Buzug

Continuous Focus Field Variation for Extending the Imaging Range in

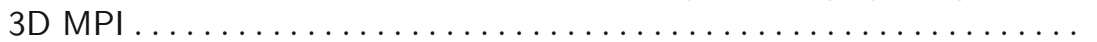

J. Rahmer, B. Gleich, J. Schmidt, C. Bontus, I. Schmale,

J. Kanzenbach, J. Borgert, O. Woywode, A. Halkola, J. Weizenecker

Third Generation X-space MPI Mouse and Rat Scanner . . . . . . . . . . . . 261 Patrick Goodwill, Laura Croft, Justin Konkle, Kuan Lu, Emine Saritas, Bo Zheng, Steven Conolly

Projection X-Space MPI Mouse Scanner . . . . . . . . . . . . . . . . . 267 Patrick Goodwill, Justin Konkle, Bo Zheng, Steven Conolly

\section{Magnetic Particle Theory}

Reconstruction of Magnetization Curve Using Magnetic Spectroscopy . . . 275 Iulian Teliban, Steffen Chemnitz, Claas Thede, Christoph Bechtold, Babak Mozooni, Eckhard Quandt, Hans-Joachim Krause 
Multiferroic Behavior of BTO-Nanoparticles

Steffen Trimper, Safa Golrokh Bahoosh, Julia M. Wesselinowa

Point Spread Function Analysis of Magnetic Particles ............. 287 Ingo Schmale, Jürgen Rahmer, Bernhard Gleich, Jörn Borgert, Jürgen Weizenecker

\section{Magneto-Relaxometry}

Spatially Resolved Measurement of Magnetic Nanoparticles Using Inhomogeneous Excitation Fields in the Linear Susceptibility Range $(<1 \mathrm{mT})$

Uwe Steinhoff, Maik Liebl, Martin Bauer, Frank Wiekhorst,

Lutz Trahms, Daniel Baumgarten, Jens Haueisen

Magnetorelaxometry for In-Vivo Quantification of Magnetic Nanoparticle Distributions after Magnetic Drug Targeting in a Rabbit Carcinoma Model ................................. 301 Frank Wiekhorst, Maik Liebl, Uwe Steinhoff, Lutz Trahms, Stefan Lyer, Stephan Dürr, Christoph Alexiou

\section{Imaging Technology and Safety Aspects}

A Control Unit for a Magnetic Particle Spectrometer ............... 309

R. Marquina-Sanchez, S. Kaufmann, M. Ryschka, T.F. Sattel,

T.M. Buzug

Optimization of Circular Current Distributions for Magnetic Field Generation in MPI: A Comparison of the Selection Field Coil and the Drive Field Coil Geometry .............................. 313 Timo F. Sattel, Marlitt Erbe, Thorsten M. Buzug

Capacitor Distortion in Magnetic Particle Imaging ................ 319 Bo Zheng, Patrick Goodwill, Wisely Yang, Steven Conolly

Safety Limits for Human-Size Magnetic Particle Imaging Systems . . . . . . 325 Emine U. Saritas, Patrick W. Goodwill, George Z. Zhang, Wenxiao Yu, Steven M. Conolly

Mouse Bed Optimized for MPI

Matthias Weber, Patrick Goodwill, Steven Conolly

Transmembrane Voltages Caused by Magnetic Fields - Numerical Study of Schematic Cell Models... 337

Alexander Kramlich, Julia Bohnert, Olaf Dössel 
Concept for a Modular Class-D Amplifier for MPI Drive Field Coils

Jonas Jockram, Oliver Woywode, Bernhard Gleich, Klaus Hoffmann

A Hybrid Filter Topology for a Reduction of High Frequency

Harmonics

J. Bergmann, K.F. Hoffmann, B. Gleich, O. Woywode

Safety Aspects for a Pre-clinical Magnetic Particle Imaging Scanner

Gael Bringout, Hanne Wojtczyk, Mandy Grüttner, Matthias Graeser,

Wiebke Tenner, Julian Hägele, Florian M. Vogt, Jörg Barkhausen,

Thorsten M. Buzug

\section{Short Contributions}

Citrate-Coated Magnetite Nanoparticles Are Highly Efficient Agents for Magnetic Labeling of Human Mesenchymal Stem Cells ...

Kristin Andreas, Jochen Ringe, Michael Sittinger, Norbert Buske

Dendronized Iron Oxides as Smart Nano-objects for Multimodal Imaging

B. Basly, G. Popa, A. Garofalo, D. Felder-Flesch, S. Begin-Colin,

C. Billotey

Superparamagnetic Dextran Coated Iron Oxide Nanoparticles (SPIO)

as Potential Markers for Tumor Cell Detection . . . . . . . . . . . . . . . 367

R. Pries, K. Lüdtke-Buzug, A. Lindemann, B. Hüsing, T.M. Buzug,

B. Wollenberg

Low Field NMR as a Tool for Neuronal Current Detection: A Feasibility Study in a Phantom. . . . . . . . . . . . . . . . . . . . . . . . . . 369 Rainer Körber, Nora Höfner, Martin Burghoff, Lutz Trahms, Jens Haueisen, Sven Martens, Gabriel Curio

Low Field Nuclear Magnetic Relaxation of Water and Brain Tissue . . . . . 371 Stefan Hartwig, Hans-Helge Albrecht, Nora Höfner, Ingo Hilschenz, Rainer Körber, Hans-Jürgen Scheer, Jens Voigt, Martin Burghoff, Lutz Trahms

In Vivo Biodistribution and Pharmacokinetics of Optimized Magnetic Particle Imaging Tracers . . . . . . . . . . . . . . . . . . . . . . . 373 Amit P. Khandhar, R. Matthew Ferguson, Kannan M. Krishnan

Imaging with Optimized Magnetite MPI Tracers 375 R. Matthew Ferguson, Amit P. Khandhar, Patrick W. Goodwill, Steven M. Conolly, Kannan M. Krishnan 
A Magnetometer Cooled with Liquid Nitrogen for the Characterization and Quantification of Magnetic Nanoparticles in Biological Samples at Room Temperature ............................ 377 Martijn Visscher, Matthias Holling, Joost Pouw, Bennie ten Haken

Uniform Magnetite Nanoparticles Larger Than 20 nm Synthesized by an Aqueous Route

Sabino Veintemillas-Verdaguer, María del Puerto Morales,

Carlos J. Serna, Manuel Andrés-Vergés, Jesús Ruiz-Cabello,

Fernando Herranz

Author Index 